Behavioral/Cognitive

\title{
Relative Valuation of Pain in Human Orbitofrontal Cortex
}

\author{
ㄱoel S. Winston, ${ }^{1,2}$ Ivo Vlaev, ${ }^{3,4}$ Ben Seymour, ${ }^{5,6}$ Nick Chater, ${ }^{4}$ and Raymond J. Dolan ${ }^{1,7}$ \\ ${ }^{1}$ Wellcome Trust Centre for Neuroimaging at UCL, London WC1N 3BG, United Kingdom, ${ }^{2}$ UCL Institute of Cognitive Neuroscience, London WC1N 3AR, \\ United Kingdom, ${ }^{3}$ Department of Surgery \& Cancer, Imperial College, London W2 1PG, United Kingdom, ${ }^{4}$ Warwick Business School, University of \\ Warwick, Coventry CV4 7AL, United Kingdom, ${ }^{5}$ Center for Information and Neural Networks, Osaka 565-0871, Japan, ${ }^{6}$ Computational and Biological \\ Learning Laboratory, Department of Engineering, Cambridge University, Cambridge CB2 1PZ, United Kingdom, and ${ }^{7}$ Max Planck UCL Centre for \\ Computational Psychiatry and Ageing Research, London WC1B 5EH, United Kingdom
}

The valuation of health-related states, including pain, is a critical issue in clinical practice, health economics, and pain neuroscience. Surprisingly the monetary value people associate with pain is highly context-dependent, with participants willing to pay more to avoid medium-level pain when presented in a context of low-intensity, rather than high-intensity, pain. Here, we ask whether context impacts upon the neural representation of pain itself, or alternatively the transformation of pain into valuation-driven behavior. While undergoing fMRI, human participants declared how much money they would be willing to pay to avoid repeated instances of painful cutaneous electrical stimuli delivered to the foot. We also implemented a contextual manipulation that involved presenting medium-level painful stimuli in blocks with either low- or high-level stimuli. We found no evidence of context-dependent activity within a conventional "pain matrix," where pain-evoked activity reflected absolute stimulus intensity. By contrast, in right lateral orbitofrontal cortex, a strong contextual dependency was evident, and here activity tracked the contextual rank of the pain. The findings are in keeping with an architecture where an absolute pain valuation system and a rank-dependent system interact to influence willing to pay to avoid pain, with context impacting value-based behavior high in a processing hierarchy. This segregated processing hints that distinct neural representations reflect sensory aspects of pain and components that are less directly nociceptive whose integration also guides pain-related actions. A dominance of the latter might account for puzzling phenomena seen in somatization disorders where perceived pain is a dominant driver of behavior.

Key words: context sensitivity; neuroeconomics; pain; subjective health complaints; valuation

\section{Introduction}

In humans, money is a universal currency against which the value of diverse outcomes can be quantified. This idea has motivated interest in the neuroscience of valuation for painful experiences (e.g., Talmi et al., 2009; Brooks et al., 2010; Park et al., 2011). These studies concentrate on neural processes that allow a trade-off between financial reward and the prospect of pain, with activity in frontostriatal valuation networks best reflecting an integrative process. Here, we address a different aspect of pain valuation, namely, its sensitivity to context.

Received April 28, 2014; revised Sept. 11, 2014; accepted Sept. 15, 2014.

Author contributions: J.S.W., I.V., B.S., N.C., and R.J.D. designed research; J.S.W. and I.V. performed research; J.S.W. and B.S. analyzed data; J.S.W., I.V., B.S., N.C., and R.J.D. wrote the paper.

J.S.W. holds a Wellcome Trust Postdoctoral Training Fellowship for MB/PhD Graduates (095939/Z/11/Z). B.S. holds a Wellcome Trust Intermediate Clinical Fellowship (097490/Z/11/Z). R.J.D. holds a Wellcome Trust Senior Investigator Award (098362/Z/12/Z). The Wellcome Trust Centre for Neuroimaging is supported by core funding from the Wellcome Trust (091593/Z/10/Z). N.C. was supported by the ERC (295917-RATIONALITY), the ESRC Network for Integrated Behavioural Science, the Leverhulme Trust (RP2012-V-022), Research Councils UK Grant EP/ K039830/1, and the Templeton Foundation. We thank the participants for their time and effort; Eric Featherstone and Oliver Josephs for help with integrating the stimulator into the MRI environment; and Benedetto De Martino, Will Penny, and Giles Story for helpful discussions.

The authors declare no competing financial interests.

This article is freely available online through the J Neurosci Author Open Choice option.

Correspondence should be addressed to Dr. Joel S. Winston, UCL Institute for Cognitive Neuroscience, 17 Queen Square, London WC1N 3AR, United Kingdom. E-mail: joel.winston@ucl.ac.uk.

DOI:10.1523/JNEUROSCI.1706-14.2014

Copyright (c) 2014 Winston et al.

This is an Open Access article distributed under the terms of the Creative Commons Attribution License (http://creativecommons.org/licenses/by/3.0), which permits unrestricted use, distribution and reproduction in any medium provided that the original work is properly attributed.
Willingness-to-pay (WTP) to avoid painful electrical stimulation is remarkably labile, showing sensitivity to local pain context, with recent experience of low-intensity pain (relative to recent experience of high-intensity pain) increasing WTP to avoid medium-intensity pain (Vlaev et al., 2009). The rank of individual painful stimuli within prolonged contexts also affects subjective intensity ratings (Watkinson et al., 2013) and brain activity associated with stimulation (Leknes et al., 2013), consistent with psychological theories of magnitude judgment (e.g., Parducci, 1965).

Unstable pain perception/valuation begs two fundamentally different interpretations. Context might modify the perception of pain itself, which is then reflected in valuations, which in turn can be taken as an accurate read-out of subjective experience. Alternatively, context might modify how pain-related information guides behavior while leaving the underlying subjective experience of pain unchanged. Neuroanatomically, if context modulates sensory processing of pain, we would expect widespread altered activity in the "pain matrix"; conversely, if context affects transformation of pain into valuations, we would expect intact stimulus representation within the "pain matrix" but altered pain representation higher up a processing hierarchy.

Neurophysiological studies concerning appetitive outcomes consistently find evidence for relative valuation (for review, see e.g., Seymour and McClure, 2008; Vlaev et al., 2011) within orbitofrontal cortex (OFC) (e.g., Tremblay and Schultz, 1999; Nieuwenhuis et al., 2005; Padoa-Schioppa and Assad, 2008; El- 

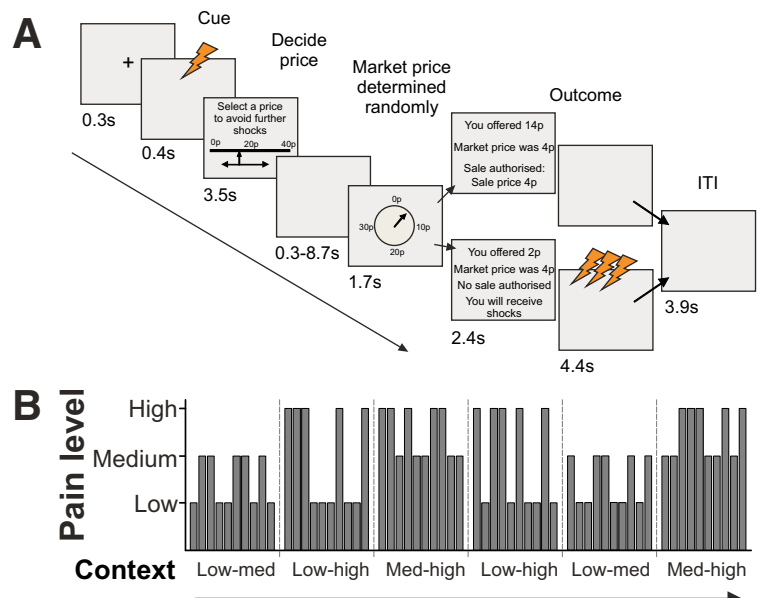

Context Low-med Low-high Med-high Low-high Low-med Med-high

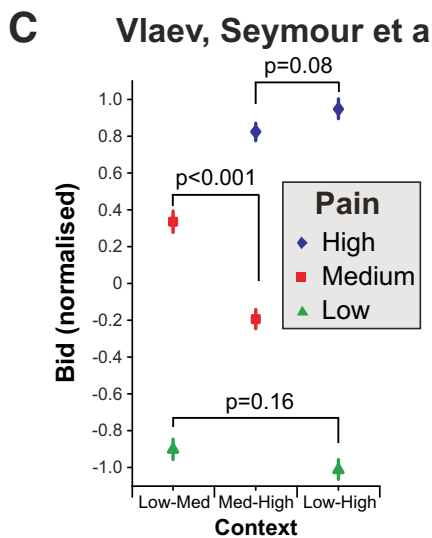

Time

Current study
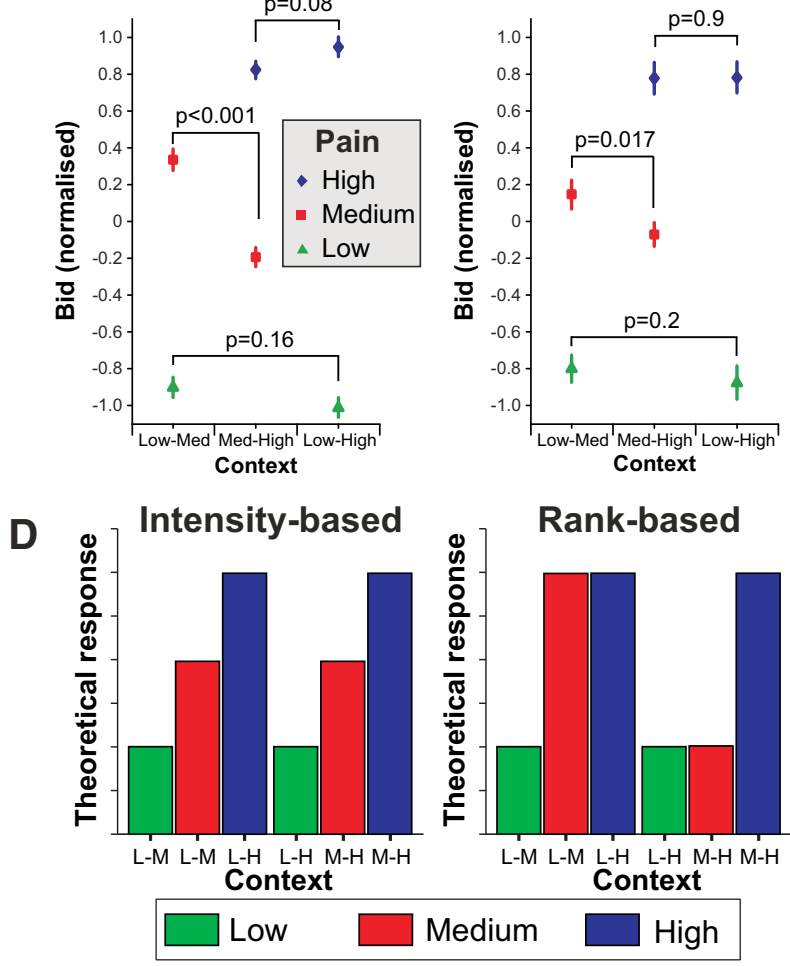

Figure 1. Task design, block structure, behavioral effects, and possible activation patterns. $A$, The components within a single trial are shown. The trial began with a warning crosshair and the endowment of 40p before a single electrical shock was delivered to inform the participant what pain level they were paying to avoid. Bids were then entered, followed by a variable delay before the market price (drawn from a uniform distribution) was announced. If participants bid more than the market price, they paid the market price and did not experience repeated shocks at the outcome phase ("pain relief"); if they bid less than the market price, they kept their entire 40p endowment but experienced the train of shocks at outcome. ITI, Intertrial interval. $\boldsymbol{B}$, Example of block structure within a single run. Blocks constituted 10 trials but contained only two of the three pain intensity levels within the experiment. The end of a block was made explicit to participants with a short break, but the existence of structure within blocks was not. C, Mean bids across participants for the six conditions from our earlier behavioral study (left) and the current dataset. Data are normalized within subject to account for the fact that the 36 subjects in Vlaev et al. (2009) constituted two groups given endowments of either 40p or 80p. Bids revealed a significant effect of context (paired pain) for the medium-level pain (replicated across the two studies) but no clear effect for the low- or high-level pain. $p$ values are different from those in the main text because of the normalization necessary for comparison across studies. Error bars indicate SEM. D, Illustrative theoretical patterns of BOLD activation for different cue-encoding mechanisms. Left, Pattern of responses encoding a pure signal of stimulus intensity. Right, Pattern that would be expected in a region encoding current stimulus rank. liott et al., 2008; Padoa-Schioppa, 2009; Kobayashi et al., 2010). However, neuroeconomic paradigms typically use stimuli for which there is no clear value signal in early sensory cortices (e.g., Plassmann et al., 2007, 2010; FitzGerald et al., 2009) and value signals might be modulated by changes in sensory-value mapping or in valuation (e.g., money is less valuable in some contexts).

Assuming that subjective experience of pain is directly linked to pain-evoked neural activity (an assumption supported by decoding of painful experience using imaging methods: Wager et al., 2013; Apkarian, 2013; and by numerous individual studies, e.g., Bornhövd et al., 2002; Coghill et al., 1999; Derbyshire et al., 1997), then measuring such activity during painful stimulation enables assessment of whether context modifies pain itself or another process (e.g., its transformation into a valuation). Here, using fMRI, we address the neural basis of relative pain valuation in a paradigm where participants experienced a single shock and expressed WTP to avoid multiple further shocks of that intensity (Fig. 1A). The experimental design (with pain sampled on every trial) ensured we could study representation of pain and value signals concurrently, asking whether contextual effects impact on the neural response to pain, or its translation into valuationrelated behavior.

\section{Materials and Methods}

Participants. Twenty-four participants were recruited into the experiment, approved by the local ethics board, via a local subject pool. Exclusion criteria were contraindications to fMRI, past medical history of significant neurological or psychiatric disease, and participation in previous experiments involving pain perception. Data from three participants were excluded from analysis: one reported moderate distress in the scanner and bid with indiscriminately high values throughout the task ( $2 / 3$ of bids at the maximum value), one due to stimulator failure (loss of electrode contact), and one due to a benign structural lesion that made normalization difficult (a large arachnoid cyst adjacent to anterior temporal lobe). The age range of the 21 included participants ( 13 females) was $20-31$ (mean $\pm \mathrm{SD}, 24.0 \pm 3.2$ years).

Painful stimulation. A Digitimer (Welwyn Garden City, UK) DS5 stimulator was used for painful stimulation. This was controlled over USB by the stimulus PC running Cogent 2000 (Laboratory of Neurobiology, UCL) implemented in MATLAB (MathWorks). A single electric shock consisted of the delivery of a series of five $10 \mathrm{~ms}$ square pulses of current to the skin, with an interval of $40 \mathrm{~ms}$ (total duration $210 \mathrm{~ms}$ ). The repeated shocks given at the outcome phase of the trial (on trials when pain relief was not bought) consisted of 10 repetitions of this stimulus each separated by a 240 ms gap.

Experimental setup. Participants were given written information and the opportunity to have any outstanding questions answered verbally by the experimenters. They lay on the scanner bed, and a pair of silver chloride EEG cap electrodes were placed $\sim 0.5 \mathrm{~cm}$ apart on the dorsum of the right foot in front of the ankle. Electrodes were filled with conductive gel and secured in place with pads and medical tape.

A standardized procedure was used to establish a threshold for maximum stimulation level and for establishing intensities for low-, medium-, and high-level pain (Vlaev et al., 2009, 2014; Seymour et al., 2012). In brief, seven intensities of stimulation ranging from $40 \%$ to $100 \%$ (at 10\% intervals) of maximal were delivered three times each in a pseudo-random order (the order of the seven intensities was random within each repetition). Participants rated these stimuli on a VAS, using an onscreen cursor controlled by four buttons: two moved the cursor to the left (one rapidly, one slowly) and two to the right. These ratings were used to estimate three parameters describing a sigmoid curve relating stimulation intensity to perceived pain level. From this fitted curve, three current intensities were estimated that corresponded to pain levels 4 ("low"), 6 ("medium"), and 8 ("high"). Where necessitated by changes in pain ratings (such as no current intensity sufficient to yield a rating of 9-10 despite the use of the preestablished maximum tolerable current), the initial thresholding and curve-fitting procedures were repeated. 
Participants then were reminded of the components of the main task by a series of text instructions onscreen. The task instructions before the main experiment included a reminder of the nature of the bidding process and the fact that the optimum price to name given the structure of the pricing was the true price that the participant would be willing to pay to avoid the prolonged painful stimulation on that trial (see also Vlaev et al., 2009). Instructions were followed by a series of four practice trials played with low-intensity painful stimulation.

The main experiment was conducted in two runs within the scanner, with a brief break to maintain participants' comfort. The study design ensured participants' decisions were incentive-compatible and were made in real-life monetary units (UK pence). Each trial took the following form (Fig. 1A): A cross-hair marked the onset of the trial and the endowment of $40 \mathrm{p}$ (i.e., $\mathfrak{£} 0.40$, equivalent to $\sim \$ 0.68$ ), and this was followed by an electric shock (the "cue"; consisting as described above of a short series of five square wave pulses of $10 \mathrm{~ms}$, which are perceived as a unitary stimulus) at one of three intensities (low, medium, or high). Participants then decided on a bid level (between $0 \mathrm{p}$ and $40 \mathrm{p}$ ) to avoid 10 further shocks at that intensity, expressing this bid level by means of an onscreen cursor moved left and right by button presses on a custom-built MRI-compatible four-button keypad interfaced with the stimulus computer via an optical USB link. Two buttons moved the cursor left (one in $4 \mathrm{p}$ decrements, one in $1 \mathrm{p}$ decrements), and two moved the cursor right. Participants chose their preferred key-mapping and held the keypad in their right hand. After a variable delay $(0-8.4 \mathrm{~s})$, a roulette wheel appeared onscreen and briefly spun, representing the random selection of a market price from a uniform distribution (1-40p). After the spinning pointer settled, onscreen text revealed the participant's bid, the market price, and the outcome (pain relief bought at the market price or no pain relief bought and the entire endowment for that trial kept; i.e., a second price auction equivalent to the Becker-DeGroot-Marschak method) (Becker et al., 1964). A further $4.4 \mathrm{~s}$ period allowed the delivery of the outcome stimulation or "pain relief" (i.e., no stimulation). After a brief intertrial interval (3.9 s), the experiment continued with the next trial. Four $5 \mathrm{~s}$ null events were inserted randomly between trials within each block.

In total, 60 trials were presented in each run (constituting 20 of each of the three levels of stimulation). Trials were presented in blocks of 10 with a brief interblock interval ( $25 \mathrm{~s}$ ), marked by onscreen text informing the participant of the break before the next block. Within a given block (and not made explicit to the participants), only two levels of stimulation were used with five trials of each level (Fig. 1B). Thus, there were three block types: low-medium, medium-high, and low-high. The order of trials within blocks was random. The order of blocks was pseudo-randomized with the constraint that each of blocks 1-3 and 4-6 within a run contained all three block types. Each run lasted $\sim 25.5 \mathrm{~min}$. At the end of the study, participants were paid according to the sums of the outcomes of

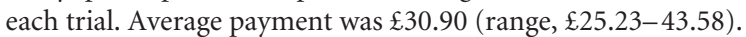

$M R$ imaging. Volunteers were scanned in a $3 \mathrm{~T}$ head scanner (Magnetom Allegra, Siemens Medical) operated with its standard head transmitreceive coil. A B0 field map was measured before the start of functional data acquisition. Functional data were acquired with a single-shot gradient-echo echoplanar image (EPI) sequence optimized for signal reconstruction in amygdala and OFC using the following imaging parameters: 42 oblique transverse slices, slice thickness $=2 \mathrm{~mm}$, gap between slices $=1 \mathrm{~mm}$, repetition time $\mathrm{TR}=2.52 \mathrm{~s}, \alpha=90^{\circ}$, echo time $=$ $30 \mathrm{~ms}$, bandwidth $=3551 \mathrm{~Hz} /$ pixel, bandwidth in phase-encoding $(\mathrm{PE})$ direction $=47.3 \mathrm{~Hz} /$ pixel, $\mathrm{PE}$ direction anterior-posterior, field of view $=192 \times 192 \mathrm{~mm}$, matrix size $64 \times 64$, with fat suppression. BOLD sensitivity losses in the OFC and the amygdala due to susceptibility artifacts were minimized by applying a $z$-shim gradient moment of -0.4 $\mathrm{mT} / \mathrm{m}^{\star} \mathrm{ms}$, a slice tilt of $-30^{\circ}$, and a positive PE gradient polarity (Weiskopf et al., 2006). EPI magnitude images were reconstructed from the complex $k$-space raw data using a generalized reconstruction method based on the measured EPI $k$-space trajectory to minimize ghosting (Josephs et al.; Proc ISMRM 8, 2000). EPI data acquisition was monitored online using a real-time reconstruction and quality assurance system (Weiskopf et al., 2007). Each functional run constituted 610 volumes with a repetition time of $2.52 \mathrm{~s}$. The first five volumes were discarded to allow for T1 equilibration. A T1-weighted structural scan was addition- ally acquired for each participant and used for normalization of functional data. For some participants, this scan was acquired in a different imaging session.

Heart rate monitoring and analysis. Heart rate was monitored using a MRI-compatible pulse oximeter (Nonin Medical) with raw plethysmograph waveforms and machine-inferred pulses recorded using Power1401 amplifier and Spike2 software (Cambridge Electronic Design), which also recorded slice times from the MRI scanner. For data analysis, plethysmograph waveforms were inspected trialwise, blinded to experimental condition and trials containing artifacts were rejected. Because of technical failures (e.g., poor oximeter placement), artifacts were common. In total, clean data were recorded from $>50 \%$ of trials for 15 subjects who were included in the analysis of heart rate data. Heart rate was calculated for each trial from the heartbeat following the cue until the end of the delay period before the announcement of the market price. Mean heart rates for each condition were entered into repeated-measures ANOVA with Greenhouse-Geisser correction for nonsphericity, and post hoc $t$ tests were used to clarify effects.

fMRI data analysis. Data analysis used SPM8 (WTCN; http://www.fil. ion.ucl.ac.uk/spm) and involved standard methods (realignment/ unwarping, normalization using parameters estimated from normalization of segmented structural images that were coregistered to EPIs and smoothing with an $8 \mathrm{~mm}$ isotropic Gaussian). Statistical inference used the GLM, implemented in SPM8 (Holmes et al., 1997). Events were characterized by $\delta$ (stick) functions at the time of onset (downsampled to 1/16th of a TR). These $\delta$ functions were convolved with the canonical hemodynamic response function to provide regressors for the GLM. Where events lasted longer than $1 \mathrm{~s}$ (rating scale use or outcome delivery), the event was modeled by a short boxcar of a length consistent with the event duration before convolution. For some event types, additional ("parametric") regressors were constructed by modulating the height of the $\delta$ function by a relevant trialwise parameter (Büchel et al., 1998).

The model included the following:

(1) Six cue types corresponding to low, medium, and high pain in each of two possible contexts, modeled as instantaneous events.

(2) A single $3.5 \mathrm{~s}$ event for the bid scale, with parametric modulators for number of button presses, distance moved, final bid value, and the interaction of context and bid value. The regressor for scale onset is correlated with the average response of the 6 regressors for cue type ( 1 above), meaning that the heights of cue responses (as shown in Figs. 2 and 3) can only be interpreted with respect to one another.

(3) An instantaneous event for the announcement of the market price, with parametric modulation by the market price, expanded up to a fourth-order polynomial term.

(4) Trial outcome (4s) for trials in which pain relief was purchased, with a parametric modulator for the cue intensity (1,2, or 3$)$.

(5) Trial outcome (4s) for trials in which the outcome shocks were delivered, with a parametric modulator for the intensity $(1,2$, or 3$)$.

We adopted a relatively high-order polynomial expansion for parametric modulator for market price where a nonlinear relationship might be expected or is of a priori interest. This approach allows a more confident delineation of the specific response profile (e.g., potentially allowing interpretation of significant loading on second-order terms as reflecting a U-shaped response profile rather than simply the presence of "nonlinearity") (for a related point, see also Winston et al., 2007). However, within this paper, the reported results reflect only responses at cue delivery, bidding value, and trial outcome (events 1, 2, 4, and 5 above). Removing the nonlinear regressors for the market price had no impact on the other reported findings.

Because of possible concerns of task-related movement with painful stimulation, several regressors were included that helped to model possible movement-related signal. The realignment parameters estimated during preprocessing were included along with their derivatives and squares (18 additional regressors). In addition, we examined the data for artifactually large scan-to-scan variance (measured on a slice-by-slice basis) and included a series of regressors with a $\delta$ function for any sequence of scans (i.e., the affected volume and those before and after) with intensity change within any single slice of $>5$ SDs. The median number 

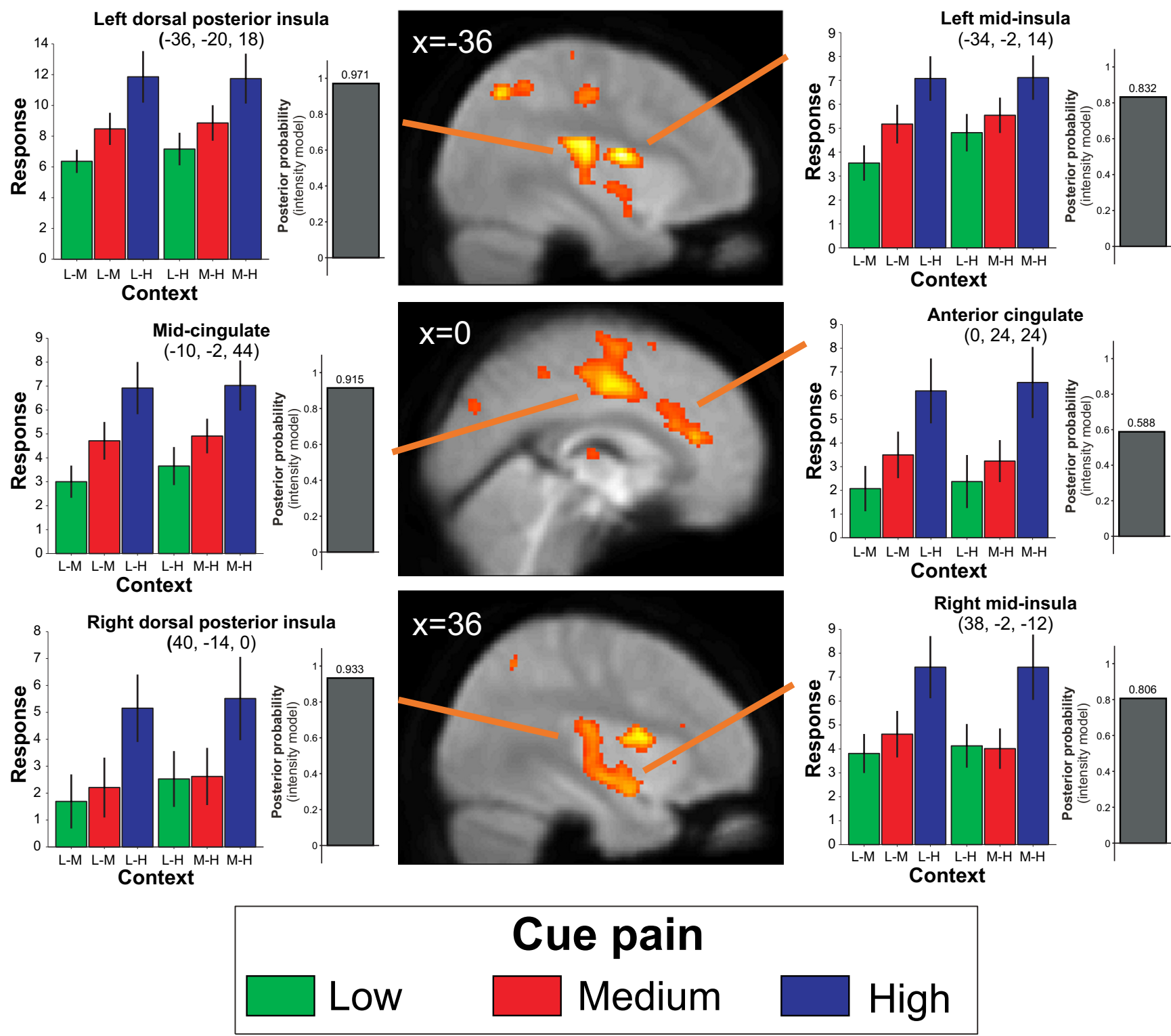

Figure 2. Responses to painful cue within pain-responsive regions are context-independent. SPM showing the main effect of pain (i.e., BOLD signal differences between the three pain levels) overlaid on the group average-normalized EPI. Threshold for display $p<0.001$ uncorrected. Colored bar charts represent across-subject mean activity levels for all conditions ( \pm SEM) for selected peak voxels (context refers to pain levels experienced within the current block: L, Low; M, medium; H, high). Bar heights are only readily interpretable relative to other conditions within the same region (see Materials and Methods). Gray bar chart represents Bayesian model selection result demonstrating that activity profiles in these regions are more consistent with intensity than rank responsiveness.

of additional regressors added to the GLM was 67 (range, 21-160). This conservative procedure essentially prevents any volumes with large signal changes from their neighbors (as would be expected by movementrelated artifact) from influencing the GLM results.

Statistical inference was at the random effects level. Contrasts of parameter estimates were conducted on single-subject GLMs, averaging across runs to generate maps of effect sizes. These formed the raw data for inference in a second-level analysis where subjects were treated as random effects. Where ANOVAs were used at the second level (for effects that depend upon multiple maps of contrasts of parameter estimates), subject effects were modeled and sphericity of errors was not assumed. Instead, iterative restrictive maximum likelihood estimation was used to estimate variance components and model coefficients (Glaser and Friston, 2003).

Bayesian model comparison was used to determine whether activity patterns in regions identified as differentially responsive to cue types best reflected intensity or rank encoding. At the random effects level, a repeated-measures ANOVA was estimated using posterior probability mapping in SPM12b. Contrasts corresponding to intensity-based and rank-based models were calculated resulting in maps of log Bayes factors for these representations. The intensity-based model corresponded to an F-contrast testing for a main effect of intensity, whereas the rank-based model corresponded to an F-contrast testing for the main effect of rank (i.e., $1 \mathrm{~s}$ where the stimulus type was the higher ranked in the current context and $0 \mathrm{~s}$ where the stimulus was the lower-ranked in the current context). The difference in these maps was compared (Penny and Ridgway, 2013), resulting in a posterior probability map indicating the evidence in favor of the alternative hypotheses. These values (expressed in terms of the posterior probability of the relevant model) are plotted in Figures 2 and 3 for peak voxels identified by the standard parametric analysis and recorded in Table 1.

All corrections for multiple comparisons (whether whole brain or for a small ROI) were based upon peak voxel statistics rather than cluster- or set-based inferences. Small volume correction (SVC) was conducted within four a priori regions of interest and based upon conservative bilateral anatomic criteria. Masks for basal ganglia, OFC and medial prefrontal cortex were generated from the Automated Anatomical Labeling reference image (Tzourio-Mazoyer et al., 2002). These were smoothed with a $4 \mathrm{~mm}$ isotropic Gaussian and thresholded (0.3) to produce binary masks of each entire re- 
gion bilaterally. Additionally, a mask for painrelated activation was derived from automated meta-analysis of 331 pain studies using Neurosynth (http://neurosynth.org) (Yarkoni et al., 2011) and used for small volume correction for pain-related activations.

Subsidiary analyses and models. We analyzed the behavioral data and constructed an additional GLM to explore whether the effects reported were robust when restricted only to those trials within a block that were experienced after both possible cue types had occurred. This indicated no substantial differences from the a priori planned analyses, which form the primary basis of our results. We also assessed whether the reported effects depended upon the specific order of expansion of parametric regressors for event Type 3 (market price), restricting the model to a third (rather than fourth) order polynomial expansion; no substantial difference in the results was seen.

Subjective Health Complaints (SHC) questionnaire. On the basis of psychological theories emphasizing the importance of dissociation between objective pathology and subjective experience in symptom reporting, we speculated that subjective health perception might relate to context-related mechanisms in acute pain perception. On a different day, within a month of the scanning session, participants were asked to complete the SHC questionnaire, a 29-item inventory of symptoms considered to be common and subjective, which may have been experienced over the past month (Eriksen et al., 1999). Seventeen of the 21 participants were successfully rerecruited. No other surveys were systematically collected on this group. Total SHC scores were log transformed as raw scores show a highly skewed distribution (e.g., Ihlebaek et al., 2002). These data were used as a regressor in exploring the relationship between the neural context sensitivity in lateral OFC and the propensity to report SHC to test the hypothesis that subjects reporting a greater burden of subjective health complaints showed enhanced neural context sensitivity during pain valuation. A mask comprising the lateral OFC activation from the contextual simple effect was used to constrain the search volume to this sole ROI for this analysis.

\section{Results}

Behavioral

Following Vlaev et al. (2009), we pre-

dicted a decrement in mean WTP for pain relief for medium-level pain in the context of medium-high blocks compared with medium-low blocks (Fig. 1C), but no effect of context on high or low pain levels. We replicated this finding ( $p=0.05$; two-tailed $t$ test on mean bids), although in the current study the effect was of smaller magnitude than reported by Vlaev et al. (2009) (a 2.5p decrement here, compare $6 \mathrm{p}$ for the 40p endowment condition in the earlier study). As in our previous study, WTP for low and high pain levels showed no significant context effects ( $p=0.094$ and $p=0.84$, respectively). The results were unchanged by a restriction of the analysis to those trials in which both trial types for the block had been experienced.

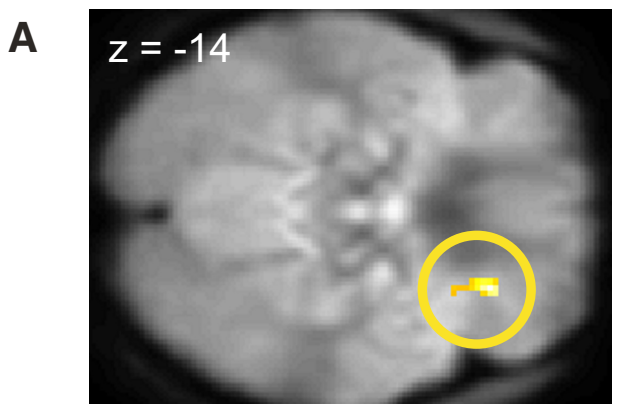

B OFC response to painful cues is

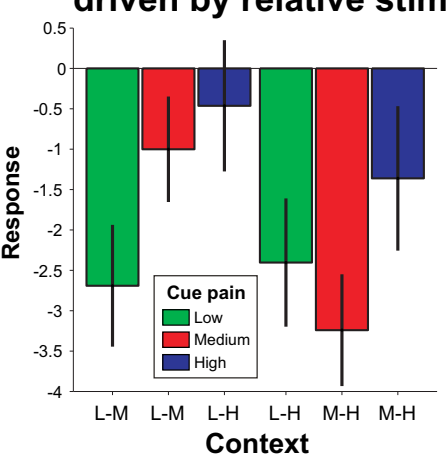

D

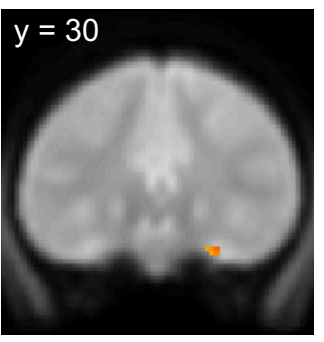

E

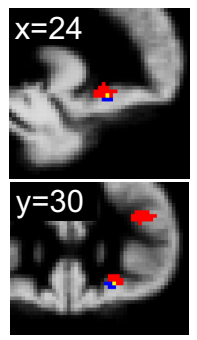

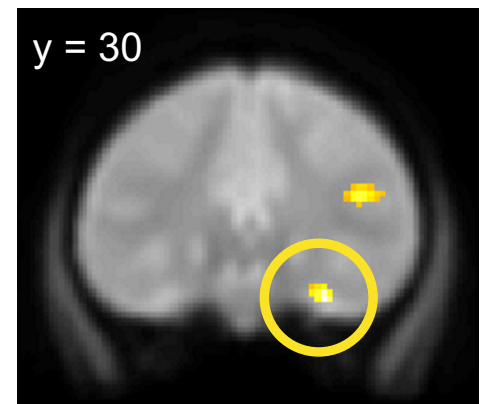

C OFC response and medical symptom burden

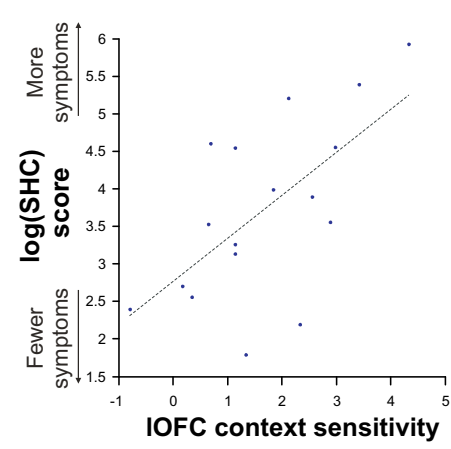

F
Figure 3. OFC responses show encoding of cue stimulus rank and bid value. $A$, SPM showing the differential effects of mediumlevel pain depending upon context (greater activity to medium level pain when in a block with low level vs high level). Display as in Figure 2. $\boldsymbol{B}$, Colored bars represent mean activity ( \pm SEM) in peak OFC voxel for all conditions, illustrating the encoding of stimulus rank in this area. Gray bar represents results of Bayesian model selection showing that activity profile in peak OFC voxel is consistent with rank-based processing. C, Correlation of context-dependent differential OFC activity to medium-level pain (i.e., medium pain in the context of low to medium pain in the context of high) and propensity to report subjective health complaints (SHC) over the past month, indexed by the SHC questionnaire. D, More medially, OFC showed encoding of bid level at the time of rank (red) encoding in lateral OFC are largely nonoverlapping with a single voxel of overlap (yellow). The area encoding bids is more medial and inferior to that encoding rank at the time of the cue. Activations thresholded at $p<0.001$ uncorrected and displayed on the group average map of gray matter density. $F$, Illustrative plot of activity relating to bid value in peak OFC voxel from a supplementary model in which events were divided into quartiles based upon bid value and the GLM refitted.

We found no evidence that bids tended to systematically drift over the time course of the study. Parameter estimates for linear models of individual bids over time did not differ from zero across participants $\left(t_{(20)}=1.3, p=0.22\right.$; for models where all pain intensities were considered separately all $p$ values $>0.13$ ). Similarly, repeated-measures ANOVA on average bids within blocks showed no effects of time regardless of whether modeled as $1 \times 12$ (for individual blocks) or $2 \times 6$ (i.e., modeling session $\times$ block) ANOVA (all $p$ values $>0.2$ ), or when assessed for trends using linear/nonlinear post hoc contrasts (linear, quadratic, and third-order effects, all $p$ values $>0.14$ ). Repeatedmeasures ANOVA with factors of stimulus position within block (1-10) and intensity revealed an effect of stimulus position 
Table 1. SPM results for main effect of cue intensity ${ }^{a}$

\begin{tabular}{|c|c|c|c|c|c|c|c|c|}
\hline Cluster & Region & k & $x$ & $y$ & $z$ & Z-score & Context effect & Model evidence \\
\hline \multirow[t]{3}{*}{1} & Left mid-insula & 1519 & -34 & -2 & 14 & $5.4^{*}$ & -0.6 & 0.83 \\
\hline & Left posterior insula & & -36 & -20 & 18 & $4.9^{*}$ & -0.6 & 0.97 \\
\hline & Left parietal operculum/posterior insula & & -54 & -32 & 24 & 4.1 & 0.0 & 0.93 \\
\hline \multirow[t]{4}{*}{2} & Left mid-cingulate gyrus & 2887 & -12 & -2 & 44 & $5.4^{*}$ & -0.3 & 0.84 \\
\hline & Left cingulate sulcus, marginal segment & & -14 & -40 & 48 & $4.8^{*}$ & 0.1 & 0.99 \\
\hline & Right mid-cingulate/posterior cingulate & & 6 & -16 & 44 & 4.3 & -0.4 & 0.97 \\
\hline & Anterior cingulate & & 0 & 34 & 18 & 4.2 & 0.7 & 0.83 \\
\hline \multirow[t]{4}{*}{3} & Right mid-insula & 1827 & 34 & 8 & 12 & $4.6^{* *}$ & 0.4 & 0.63 \\
\hline & Right inferior mid-insula & & 38 & -2 & -12 & $4.5^{* *}$ & 0.9 & 0.81 \\
\hline & Right posterior insula & & 40 & -14 & 0 & $4.5^{* *}$ & -0.5 & 0.93 \\
\hline & Right anterior insula & & 48 & 16 & -6 & 3.9 & 0.1 & 0.83 \\
\hline 4 & Right inferior frontal gyrus & 166 & 42 & 24 & 16 & $4.6^{* *}$ & 2.0 & 0.99 \\
\hline 5 & Left intraparietal sulcus & 180 & -36 & -62 & 44 & 4.4 & 0.1 & $>0.99$ \\
\hline 6 & Left middle temporal gyrus & 94 & -56 & -64 & 4 & 4.0 & 0.9 & 0.99 \\
\hline 7 & Left postcentral gyrus & 86 & -30 & -28 & 58 & 4.0 & -0.4 & 0.88 \\
\hline 8 & Right thalamus & 55 & 8 & -2 & 6 & 3.5 & 0.8 & 0.75 \\
\hline 9 & Right middle frontal gyrus & 29 & 50 & 8 & 46 & 3.5 & -0.8 & 0.99 \\
\hline 10 & Thalamus & 21 & 0 & -16 & 10 & 3.3 & 0.3 & 0.77 \\
\hline \multirow[t]{2}{*}{$11^{b}$} & Right parieto-occipital fissure & 499 & 8 & -74 & 36 & 4.5 & 2.1 & 0.99 \\
\hline & Left parieto-occipital fissure & & -10 & -74 & 34 & 4.4 & 3.3 & 0.99 \\
\hline $12^{b}$ & Left central sulcus & 83 & -34 & -20 & 42 & 3.8 & 0.9 & 0.50 \\
\hline $13^{b}$ & Left postcentral gyrus & 25 & -58 & -40 & 46 & 3.8 & -0.8 & 0.72 \\
\hline $14^{b}$ & Left precentral gyrus & 30 & -40 & -2 & 50 & 3.7 & 0.4 & 0.55 \\
\hline $15^{b}$ & Right superior temporal sulcus & 37 & 56 & -52 & 12 & 3.6 & 0.2 & 0.98 \\
\hline $16^{b}$ & Right intraparietal sulcus & 37 & 36 & -54 & 48 & 3.6 & 0.3 & 0.96 \\
\hline $17^{b}$ & Right superior precentral sulcus & 52 & 46 & -6 & 50 & 3.4 & -0.2 & 0.90 \\
\hline $18^{b}$ & Left anterior calcarine sulcus & 36 & -14 & -58 & 4 & 3.4 & 2.4 & 0.81 \\
\hline $19^{b}$ & Right postcentral gyrus & 29 & 16 & -34 & 70 & 3.4 & -1.0 & 0.96 \\
\hline
\end{tabular}

${ }^{a}$ SPMs inspected at $p<0.001$ uncorrected, minimum cluster size of 20 contiguous voxels. Peaks of clusters 1-10 fall within the mask used for SVC for this contrast (derived from a meta-analysis of pain-related studies).

${ }^{b}$ Remaining clusters fell outside this mask. Cluster size ( $k$ ) refers to total cluster size (i.e., including voxels outside the mask where the peak falls within). "Context effect" is the z-score for the simple effect of context on medium intensity cues (medium in the context of low to medium in the context of high). "Model evidence" refers to a random effects model comparison of intensity-processing and rank-processing models, reflecting the posterior probability favoring an intensity-processing model over a rank-processing model. No peak voxels within the key regions of interest show a significant context effect, and no region shows a posterior probability favoring the rank-processing model.

${ }^{*} p<0.05$, corrected for multiple comparisons across entire brain volume.

${ }^{* *} p<0.05$, corrected for multiple comparisons across small volume of interest.

$\left(F_{(5.2,104.7)}=4.1, p<0.01\right)$ in addition to the expected main effect of intensity $\left(F_{(1.2,24.4)}=72.3, p<0.001\right)$ but no interaction between stimulus position and intensity $\left(F_{(7.1,141.8)}=0.6, p=0.75\right)$. Inspection of mean bids showed that the first stimulus of the block attracted a lower bid than other stimuli with no difference between the remaining stimuli $\left(F_{(5.3,105.1)}=0.5, p=0.78\right)$.

Analysis of heart rate responses available from a subset of participants $(n=15)$ showed a main effect of stimulation intensity $\left(F_{(1.6,23.0)}=10.6, p=0.001\right)$, driven by faster responses to high intensity cues than medium $\left(t_{(14)}=-3.4, p<0.005\right)$ or low $\left(t_{(14)}=-3.7, p<0.005\right)$. There was a simple effect of context on heart rate following medium intensity cues $\left(t_{(14)}=-2.4, p<\right.$ $0.05)$ but no evidence of contextual effects for low- or highintensity cues (low: $t_{(14)}=-1.3, p=0.20$; high: $t_{(14)}=0.3, p=$ $0.78)$. The specific pattern of cardiac responses was distinct from the pattern of fMRI responses in either pain-responsive regions or OFC (medium level cues were associated with faster heart rate responses in the context of high-intensity than low-intensity trials), suggesting that arousal effects due to painful stimulation are unlikely to explain regional fMRI effects.

\section{fMRI}

Effects at cue

The presentation of a single painful cue, indicating pain intensity on the current trial, was the key time point of interest in the fMRI analysis. Theoretical patterns of activity corresponding to intensity- or rank-based processing of cue intensity are shown for illustration in Figure 1D. An F-contrast for the main effect of intensity revealed BOLD differences across a set of regions, usually referred to as the "pain matrix" (but see Iannetti and Mouraux, 2010), including cingulate cortex, bilateral insula, left S1, left S2, and thalamus (Table 1). Inspection of parameter estimates revealed that these areas manifest increasing BOLD response with increasing pain intensity. Responses within these areas showed no modulation by context (Fig. 2; Table 1).

Our critical comparison was that of medium pain level cues across the two contexts (medium in context of low or high pain, respectively). This revealed a significant difference in lateral OFC $(x, y, z=26,30,-14 ; Z=4.7, p<0.01$ family-wise error [FWE] with SVC; Figure $3 A, B)$. Here, the profile of activity corresponded to the rank of the current stimulus within the current context, rather than purely to intensity. No activation passed statistical correction for multiple comparisons across the whole brain; and within a priori ROIs other than OFC, no activation passed statistical correction for multiple comparisons across the volume of the ROI (right dorsolateral PFC $x, y, z=40,32,22 ; Z=$ 3.6; rostral anterior cingulate $x, y, z=8,28,26 ; Z=3.2$; both $p<$ 0.001 uncorrected, $p>0.5$ after SVC for ROI of pain-responsive regions derived from meta-analysis).

The results of analyses of effects of intensity and context on responses to the cues were not substantively changed by restriction of the analysis to those trials alone in which both trial types for the block had been experienced.

To formally compare patterns of activity, we implemented a Bayesian model comparison, using the Savage-Dickey-Taylor ap- 
proximation to the Bayes factor (Penny and Ridgway, 2013). At the random effects level, log Bayes factor maps were computed for two contrasts: (1) the main effect of intensity (i.e., any pattern of differences between cue intensity level, collapsed across context); and (2) the main effect of rank (positive weights on cues that represent the higher ranked cue within the current context and negative weights on cues representing the lower ranked cue in the current context). We inspected the subtraction of these maps at the peak coordinates above to assess the relative model evidence for rank-based or intensity-based processing in each of the areas highlighted. In sensory, insula, and cingulate cortices, there was greater model evidence for intensity-based processing than rank-based (Fig. 2; Table 1). By contrast, in the lateral OFC model, evidence strongly favored a rank-based as opposed to an intensity model (Fig. 3).

\section{Bid-related activity}

The analysis above showed that activity in set of brain regions, the "pain matrix," reflects intensity of the cue pain regardless of context, whereas activity in the lateral OFC more clearly reflects the rank of pain intensity within the current context. However, from a purely behavioral perspective, the economic value of pain reported by participants combines information about both absolute intensity and rank (Fig. 1C). To reveal regions encoding WTP, we examined BOLD activity correlating with bid level at the time of decision (including in the GLM aspects directly pertaining to motor responses, such as the distance moved along the bidding scale and number of button presses). We observed a negative correlation with bid level in right $\mathrm{OFC}$, more medial to the activation related to cue stimulus rank $(x, y, z=20,28,-14 ; Z=3.9, p<0.05$ FWE with SVC; Fig. $3 D, E)$. Additionally, activity was seen in visual regions, presumably reflecting visual attention to the extremes of the scale (negative correlation with bids $x, y, z=-6,-78,4 ; Z=5.7 ; p<0.05 \mathrm{FWE}$; positive correlation with bids: $x, y, z=16,-76,10 ; Z=4.1 ; p<$ 0.001 uncorrected). There was no bid-by-context interaction, even at a liberal threshold ( $p<0.05$ uncorrected) at any of the peaks highlighted above, nor were there any other areas at conventional significance levels.

\section{Relationship between context effects in OFC and symptom reporting}

The relative encoding of the same medium level stimulus in OFC could be construed as a failure of accurate interoceptive coding of some aspect of pain valuation within this region. It is of interest that a dissociation between absolute and perceived interoceptive signals is a hallmark of somatization, whereby subjective experiences become health-related complaints (Barsky and Wyshak, 1990). Therefore, we undertook an exploratory analysis, regressing magnitude of the context effect for medium-level cues in OFC against a measure of propensity to report subjective health complaints, the SHC. Greater lateral OFC context effects were associated with expression of greater symptom burden $(x, y, z=24,30$, $-10 ; Z=2.7 ; p<0.05$ SVC for search region within lateral OFC; Fig. $3 C$ ), implying that neural context sensitivity for pain in lateral OFC and the propensity to report SHCs might be related.

\section{Effects related to outcome}

There were main effects of pain relief at outcome (relative to pain) in bilateral posterior occipitoparietal cortex $(x, y, z=14$, $-96,14 ; Z=5.8 ; x, y, z=32,-64,30 ; Z=5.1 ; x, y, z=-34$, $-68,54 ; Z=5.7 ; x, y, z=-26,-76,28 ; Z=5.5$; all $p<0.05$ FWE) and left ventral occipital cortex $(x, y, z=-42,-72,-14$; $Z=5.0 ; p<0.05 \mathrm{FWE})$. Activation was evident in left anterior
OFC and ventromedial PFC $(x, y, z=-28,58,-4 ; Z=4.3 ; x, y$, $z=2,60,-2 ; Z=4.1 ; x, y, z=10,36,-12 ; Z=4.1 ; p<0.05$ FWE after SVC for bilateral OFC). Table 2 lists all clusters at $p<$ 0.001 uncorrected. Activity at peak voxels did not appear to be significantly modulated by the intensity of the painful stimulus that was avoided (Table 2).

The converse contrast of pain $>$ relief was associated with widespread activation in expected areas, including bilateral insula, left S1 and bilateral S2, cingulate cortex, and thalamus (Table 2). Ventral pallidum additionally showed strong differential responses. Most peak voxels showed activation profiles that were modulated by pain intensity (Table 2 ).

\section{Discussion}

How humans value pain is a critical question spanning neuroscience, psychology, economics, and clinical practice. Here, we attempted to resolve the puzzle of relative judgment in pain valuation within a neuroeconomic framework, exploiting a paradigm that ensured participants' decisions regarding valuation of pain were incentive-compatible (as bids within a BeckerDeGroot-Marschak auction should reflect true private valuations). We replicated our previous behavioral findings that pain valuation is context-sensitive (Vlaev et al., 2009; Kurniawan et al., 2010) but now elucidate a neural basis of this effect, expressed in lateral OFC response profile. This speaks to the strength of a neuroeconomic approach, where neural data provide an enriched perspective on apparent anomalies in human decision making that neoclassical economic theory struggles to accommodate (Camerer, 2007; Delgado et al., 2008; Glimcher, 2011).

Our neural data afforded an approach to a research question that is difficult to reliably answer behaviorally: namely, does context alter nociception itself or modify how nociceptive information is used to guide behavior? Our results support the latter hypothesis. We find that, whereas brain regions classically associated with nociception (those within the "pain matrix") reflect absolute stimulus intensity, the OFC (an important region in representation of instrumental value) reflected the relative rank of pain (in relation to other pains in the same block of trials). Observed behavior reflects a combination of both dimensions; and consistent with this, we found evidence for a representation of overall economic value, reflecting a combination of intensity and rank, at the time of valuation within more medial OFC.

The observation of strongly contextual responses in OFC is a neural signature of psychological (e.g., Stewart et al., 2005) and economic (e.g., Barberis et al., 2006) models of choice behavior that emphasize the importance of short-term stimulus histories in judgment and decision making. Intriguingly, this finding suggests that these short-term phenomena in judgment emerge from neural mechanisms more tightly linked to executive/effector rather than perceptual systems. We speculate that alternative schemes for range normalization could account for the differences between OFC encoding rank and the regions within the "pain matrix" that more faithfully encode intensity within our paradigm (for recent perspectives on value normalization and impacts on value-based decisionmaking, see Rangel and Clithero, 2012; De Martino and Louie, 2014). This possibility can explain why value-based decision (engaging OFC) is more susceptible to contextual effects than classical economic theory would predict (De Martino et al., 2006; Soltani et al., 2012) (e.g., decoy-dependent choice and framing effects). Although OFC does not always show reference-dependent value signals (e.g., De Martino et al., 2009), the specific time course of stimuli is likely an important determinant of value normalization (e.g., PadoaSchioppa, 2009; Kobayashi et al., 2010). 
Table 2. SPM results for main effect of outcome type (pain/relief) ${ }^{a}$

\begin{tabular}{|c|c|c|c|c|c|c|c|}
\hline Cluster & Region & $k$ & $x$ & $y$ & $z$ & Main effect (Z) & Intensity effect (Z) \\
\hline \multicolumn{8}{|c|}{ Pain $>$ relief } \\
\hline \multirow[t]{5}{*}{1} & Left insula, parietal/frontal operculum & 9663 & -40 & -10 & -2 & $6.7^{*}$ & 3.1 \\
\hline & & & -52 & -28 & 18 & $6.6^{*}$ & 3.2 \\
\hline & & & -44 & -32 & 20 & $6.6^{*}$ & 2.6 \\
\hline & & & -58 & 4 & 4 & $6.1^{*}$ & 3.8 \\
\hline & Right ventral pallidum & & 18 & 2 & -4 & $4.7^{*}$ & 1.4 \\
\hline \multirow[t]{3}{*}{2} & Right insula, parietal/frontal operculum & 6093 & 62 & -30 & 24 & $6.6^{*}$ & 4.6 \\
\hline & & & 46 & 0 & 4 & $6.4^{*}$ & 3.7 \\
\hline & & & 52 & 6 & 4 & $6.2^{*}$ & 3.7 \\
\hline \multirow[t]{4}{*}{3} & Left medial postcentral gyrus & 4558 & -14 & -44 & 58 & $5.5^{*}$ & 3.2 \\
\hline & Left medial superior frontal gyrus & & -8 & -8 & 66 & $5.5^{*}$ & 4.5 \\
\hline & Bilateral mid-cingulate/anterior cingulate gyrus & & 10 & 12 & 38 & $5.4^{*}$ & 3.3 \\
\hline & & & -6 & -4 & 42 & $5.3^{*}$ & 4.6 \\
\hline 4 & Right middle frontal gyrus & 122 & 52 & -2 & 48 & $4.4^{* *}$ & 3.2 \\
\hline 5 & Right middle frontal gyrus & 90 & 28 & 44 & 24 & $4.3^{* *}$ & 1.0 \\
\hline $6^{b}$ & Right medial postcentral gyrus & 66 & 18 & -44 & 54 & 4.2 & 2.6 \\
\hline 7 & Right cerebellum & 62 & 30 & -58 & -28 & 4.0 & 2.7 \\
\hline 8 & Left cerebellum & 67 & -40 & -58 & -32 & 3.9 & 2.3 \\
\hline $9^{b}$ & Posterior hippocampus & 124 & -28 & -48 & 0 & 3.9 & -0.8 \\
\hline $10^{b}$ & Left cerebellum & 43 & -24 & -64 & -24 & 3.8 & 3.1 \\
\hline 11 & Left middle frontal gyrus & 46 & -26 & 30 & 24 & 3.6 & -0.3 \\
\hline $12^{b}$ & Right posterior hippocampus & 42 & 26 & -46 & 8 & 3.6 & -1.7 \\
\hline $13^{b}$ & Right lingual gyrus & 32 & 28 & -46 & -8 & 3.5 & 0.5 \\
\hline \multicolumn{8}{|c|}{ Relief $>$ pain } \\
\hline \multirow[t]{3}{*}{1} & Right occipital pole & 10181 & 14 & -96 & 14 & $5.8^{*}$ & 1.7 \\
\hline & Left superior parietal gyrus & & -34 & -68 & 54 & $5.7^{*}$ & -1.0 \\
\hline & Left intraparietal sulcus & & -26 & -76 & 28 & $5.5^{*}$ & 0.4 \\
\hline 2 & Left anterior superior temporal sulcus & 32 & -52 & 6 & -20 & $5.2^{*}$ & 0.7 \\
\hline \multirow[t]{2}{*}{3} & Left lateral fusiform gyrus & 1682 & -42 & -72 & -14 & $5.0^{*}$ & -0.8 \\
\hline & Left superior temporal sulcus & & -56 & -36 & 0 & $4.8^{*}$ & -0.2 \\
\hline \multirow[t]{2}{*}{4} & Right middle frontal gyrus & 912 & 30 & 14 & 60 & $5.0^{*}$ & -0.5 \\
\hline & Right superior frontal gyrus & & 18 & 36 & 50 & 4.5 & -1.0 \\
\hline 5 & Right middle temporal gyrus & 194 & 62 & -2 & -18 & 4.5 & 0.5 \\
\hline 6 & Left frontomarginal sulcus & 27 & -28 & 58 & -4 & $4.3^{* *}$ & 0.0 \\
\hline \multirow[t]{3}{*}{7} & Ventral medial prefrontal cortex & 228 & 2 & 60 & -2 & $4.1^{* *}$ & 0.6 \\
\hline & & & 10 & 56 & -8 & 3.8 & -0.9 \\
\hline & & & -6 & 48 & -6 & 3.7 & 0.7 \\
\hline 8 & Ventral medial prefrontal cortex & 25 & 10 & 36 & -12 & $4.1^{* *}$ & 0.3 \\
\hline \multirow[t]{2}{*}{9} & Left inferior frontal gyrus & 783 & -38 & 18 & 52 & 4.1 & -0.7 \\
\hline & Left middle frontal gyrus & & -54 & 22 & 20 & 4.1 & -0.6 \\
\hline 10 & Left anterior middle temporal gyrus & 30 & 54 & 10 & -28 & 4.0 & 0.6 \\
\hline 11 & Right inferior temporal gyrus & 41 & 56 & -40 & -8 & 3.9 & 0.3 \\
\hline 12 & Anterior cingulate & 30 & 14 & 42 & 14 & 3.5 & 0.6 \\
\hline 13 & Left superior frontal gyrus & 24 & -12 & 62 & 20 & 3.4 & 2.1 \\
\hline 14 & Left superior frontal gyrus & 23 & -10 & 34 & 54 & 3.4 & 0.2 \\
\hline 15 & Cuneus & 23 & -4 & -64 & 28 & 3.3 & 0.7 \\
\hline
\end{tabular}

${ }^{a}$ SPMs inspected at $p<0.001$ uncorrected, minimum cluster size of 20 contiguous voxels. SVC for pain $>$ relief is for the volume of a meta-analytically derived mask of pain-related activations. SVC for relief $>$ pain is for a mask of bilateral 0 FC.

${ }^{b}$ Peaks outside the mask of pain-related activations used for SVC. "Intensity effect" records the Z-value for a contrast looking for positive linear effects of pain level at the time of outcome for the relevant outcome (i.e., restricted to pain or relief). Many of the peak voxels for the contrast pain $>$ relief show modulation by stimulus intensity, whereas this is not the case for peak voxels for the contrast relief $>$ pain (where there is no painful stimulation at the outcome stage). ${ }^{*} p<0.05$, corrected for multiple comparisons across entire brain volume.

${ }^{* *} p<0.05$, corrected for multiple comparisons across small volume of interest.

The lateral OFC region that expressed a contextual modulation of its response is widely implicated in emotional and decision-making behaviors (Schoenbaum et al., 2011; Grabenhorst and Rolls, 2011) and receives highly integrated sensory information from all modalities, including somatosensory regions and the insular cortex (Carmichael and Price, 1995a,b). Although there is an ongoing debate about its precise role in emotional learning and decision making, lateral OFC is widely implicated in responding appropriately to stimuli whose outcome values are altered (Schoenbaum et al., 2011; Rudebeck et al., 2013) (e.g., by sensory-specific satiety). Our observation that both contextual modulation (i.e., stimulus rank sensitivity) and bidding behavior (i.e., overall stimulus value) are represented in OFC is consistent with these observations, but we now extend these into the domain of the relative coding of aversive experience.

Unlike central nodes within the "pain matrix," OFC showed modulation of its response to the same painful stimulus as a function of context. Given that many psychological theories of somatization posit a disconnection between objective sensory signals in the body and subjective experience (e.g., Barsky and Wyshak, 1990), we asked whether this contextual modulation is linked to the experience of subjective health complaints (SHC). This exploratory analysis led to the intriguing observation that the magnitude of contextual differentiation between the same 
medium-intensity stimulus by OFC correlated with participants' propensity to report SHC. Although we are cautious about the interpretation of this exploratory analysis, one implication is that susceptibility to contextual effects for pain could partially underpin some subjective medical symptoms (e.g., by causing behavior to be more influenced by relative than absolute aspects of interoceptive perceptual experiences, thus increasing the disconnection between objective pathology and perceived symptomatology). It will be interesting to address this question in future studies, particularly given that pain-based symptoms represent a substantial burden of medically unexplained disease (Reid et al., 2001).

Participants' bid values were reflected in activity within a distinct portion of OFC. Critically, there was no evidence for an interaction between bid and context within this area (or any other representing bid value). This suggests that the contextual influence over bids is best construed as resulting from a ranking process implemented in lateral OFC, rather than an altered utility function for money under different contexts. The relationship between bids and BOLD activation in OFC was negative, consistent with this region representing value (higher bids reflect greater aversiveness). The finding of overall valuation (indexed by bids) in close proximity to adaptive valuation has some similarity to single-unit responses reported by Kobayashi et al. (2010). There, subpopulations of value-responsive lateral OFC neurons exhibit range normalization to a manipulation of reward variance, with other neurons showing responses to overall value without normalization. Interestingly, they demonstrated normalization of responses to blocks of similar length to those adopted in the current study (using random block lengths of 4-13 trials), although this pattern was more widespread with longer blocks.

The contextual manipulation and task used here were rather different from those adopted in other neurophysiological studies, which have concentrated largely on pain perception rather than valuation. Interestingly, other studies have not generally reported modulation of orbitofrontal responses due to contextual manipulation of pain perception. Placebo analgesia is characterized by modulation of activity within a network of lateral and medial prefrontal regions, anterior cingulated, and periacqueductal gray matter (Amanzio et al., 2013), and extends even to the spinal cord (Eippert et al., 2009). Manipulation of pain perception by emotional context is associated with altered activity in anterior insula (Ploner et al., 2011), and a recent study using prolonged stimulus-driven context showed widespread changes in activity in ventral medial prefrontal cortex and periacqueductal gray matter related to relative relief (Leknes et al., 2013). Comparison with this latter study is particularly illuminating; a similar manipulation of stimulus rank was used by Leknes et al. (2013) as in the current study but over much longer contexts (10 min blocks with a 5 min break). Effects of this manipulation were seen within core regions of the "pain matrix," such as anterior cingulate and insula, whereas these regions showed no significant context effects over the substantially shorter blocks adopted here. These apparent differences in the neural bases for distinct types of contextual modulations of pain perception or valuation suggest that there are multiple potential mechanisms by which nociception and cognition interact to produce pain-related behaviors. It seems noteworthy that similar regions within OFC demonstrate differential activation in response to other cognitive manipulations of pain: externally versus self-provoked pain (Wiech et al. 2006), placebo and opiod analgesia (Petrovic et al. (2002), and the magnitude of anticipated placebo analgesia (Wager et al., 2004; Wiech et al., 2008).

Interestingly, we found that successful avoidance of pain was associated with activation at the outcome phase of the trial in ventromedial and ventrolateral prefrontal cortex. This is consistent with previous findings that relief from tonic pain is associated with activation in similar regions (e.g., Petrovic et al., 2002; Wager et al., 2004; Seymour et al., 2005).

Although stimulus rank appeared to be the dominant heuristic encoded in lateral OFC and influencing bidding behavior, it seems likely that other heuristics might influence bids under different circumstances. The paradigm we adopted fairly selectively isolates rank rather than other characteristics, such as stimulus contrast (evidenced by the absence of context effects on high- and low-intensity stimuli here and in Vlaev et al., 2009). Within the current study (Fig. 2) and consistent with previous results (e.g., Bornhövd et al., 2002), some regions within the pain matrix show nonlinear (though not rank-driven) relationships with the underlying stimulus, which could theoretically form the basis of alternative biases in valuation. The mechanisms by which other stimulus-driven heuristics might influence pain valuation will be of interest in future studies.

Together, our results indicate a dissociation between painresponsive regions that faithfully represent the intensity of a painful stimulus and OFC whose activity reflects alternative constructs, such as local stimulus rank and overall value. We suggest that these latter regions contribute to decision-making about pain that appears irrational but actually reflects the integration of competing influences with alternative valuation metrics. Our observation that neural context sensitivity is predictive of symptom-reporting propensity strengthens our confidence that improved understanding of the neuroeconomics of pain can provide insight into clinical conundrums that surround painrelated behavior and symptomatology.

\section{References}

Amanzio M, Benedetti F, Porro CA, Palermo S, Cauda F (2013) Activation likelihood estimation meta-analysis of brain correlates of placebo analgesia in human experimental pain. Hum Brain Mapp 34:738-752. CrossRef Medline

Apkarian AV (2013) A brain signature for acute pain. Trends Cogn Sci 17: 309-310. CrossRef Medline

Barberis N, Huang M, Thaler RH (2006) Individual preferences, monetary gambles, and stock market participation: a case for narrow framing. Am Econ Rev 96:1069-1090. CrossRef

Barsky AJ, Wyshak G (1990) Hypochondriasis and somatosensory amplification. Br J Psychiatry 157:404-409. CrossRef Medline

Becker GM, DeGroot MH, Marschak J (1964) Measuring utility by a singleresponse sequential method. Behav Sci 9:226-232. CrossRef Medline

Bornhövd K, Quante M, Glauche V, Bromm B, Weiller C, Büchel C (2002) Painful stimuli evoke different stimulus-response functions in the amygdala, prefrontal, insula and somatosensory cortex: a single-trial fMRI study. Brain 125:1326-1336. CrossRef Medline

Brooks AM, Pammi VS, Noussair C, Capra CM, Engelmann JB, Berns GS (2010) From bad to worse: striatal coding of the relative value of painful decisions. Front Neurosci 4:176. CrossRef Medline

Büchel C, Holmes AP, Rees G, Friston KJ (1998) Characterizing stimulusresponse functions using nonlinear regressors in parametric fMRI experiments. Neuroimage 8:140-148. CrossRef Medline

Camerer CF (2007) Neuroeconomics: using neuroscience to make economic predictions. Econ J 117:C26-C42. CrossRef

Carmichael ST, Price JL (1995a) Sensory and premotor connections of the orbital and medial prefrontal cortex of macaque monkeys. J Comp Neurol 363:642-664. CrossRef Medline

Carmichael ST, Price JL (1995b) Limbic connections of the orbital and medial prefrontal cortex in macaque monkeys. J Comp Neurol 363:615-641. CrossRef Medline

Coghill RC, Sang CN, Maisog JM, Iadarola MJ (1999) Pain intensity processing within the human brain: a bilateral, distributed mechanism. J Neurophysiol 82:1934-1943. Medline

Delgado MR, Schotter A, Ozbay EY, Phelps EA (2008) Understanding overbidding: using the neural circuitry of reward to design economic auctions. Science 321:1849-1852. CrossRef Medline 
De Martino B, Louie K (2014) The neurobiology of context-dependent valuation and choice. In: Neuroeconomics: decision making and the brain, Ed 2 (Glimcher PW, Fehr E, eds). New York: Academic.

De Martino B, Kumaran D, Seymour B, Dolan RJ (2006) Frames, biases, and rational decision-making in the human brain. Science 313:684-687. CrossRef Medline

De Martino B, Kumaran D, Holt B, Dolan RJ (2009) The neurobiology of reference-dependent value computations. J Neurosci 29:3833-3842. CrossRef Medline

Derbyshire SW, Jones AK, Gyulai F, Clark S, Townsend D, Firestone LL (1997) Pain processing during three levels of noxious stimulation produces differential patterns of central activity. Pain 73:431-445. CrossRef Medline

Eippert F, Finsterbusch J, Bingel U, Büchel C (2009) Direct evidence for spinal cord involvement in placebo analgesia. Science 326:404. CrossRef Medline

Elliott R, Agnew Z, Deakin JF (2008) Medial orbitofrontal cortex codes relative rather than absolute value of financial rewards in humans. Eur J Neurosci 27:2213-2218. CrossRef Medline

Eriksen HR, Ihlebaek C, Ursin H (1999) A scoring system for subjective health complaints (SHC). Scand J Public Health 27:63-72. CrossRef Medline

FitzGerald TH, Seymour B, Dolan RJ (2009) The role of human orbitofrontal cortex in value comparison for incommensurable objects. J Neurosci 29:8388-8395. CrossRef Medline

Glaser DE, Friston KJ (2003) Variance components. In: Human brain function, Ed 2 (Frackowiak RSJ, Friston KJ, Frith CD, Dolan RJ, Price CJ, Zeki S, Ashburner J, Penny WD, eds), pp 781-791. San Diego: Academic.

Glimcher PW (2011) Foundations of neuroeconomic analysis. New York: Oxford UP.

Grabenhorst F, Rolls ET (2011) Value, pleasure and choice in the ventral prefrontal cortex. Trends Cogn Sci 15:56-67. CrossRef Medline

Holmes AP, Poline JP, Friston KJ (1997) Characterizing brain images with the general linear model. In: Human brain function, Ed 1 (Frackowiak RSJ, Friston KJ, Frith CD, Dolan RJ, Mazziotta JC, eds), pp 59-84. San Diego: Academic.

Iannetti GD, Mouraux A (2010) From the neuromatrix to the pain matrix (and back). Exp Brain Res 205:1-12. CrossRef Medline

Ihlebaek C, Eriksen HR, Ursin H (2002) Prevalence of subjective health complaints (SHC) in Norway. Scand J Public Health 30:20-29. CrossRef Medline

Josephs O, Deichmann R, Turner R (2000) Trajectory Measurement and generalized reconstruction in rectilinear EPI. Pro Int Soc Magn Reson Med 8:1517.

Kobayashi S, Pinto de Carvalho O, Schultz W (2010) Adaptation of reward sensitivity in orbitofrontal neurons. J Neurosci 30:534-544. CrossRef Medline

Kurniawan IT, Seymour B, Vlaev I, Trommershäuser J, Dolan RJ, Chater N (2010) Pain relativity in motor control. Psychol Sci 21:840-847. CrossRef Medline

Leknes S, Berna C, Lee MC, Snyder GD, Biele G, Tracey I (2013) The importance of context: when relative relief renders pain pleasant. Pain 154: 402-410. CrossRef Medline

Nieuwenhuis S, Heslenfeld DJ, von Geusau NJ, Mars RB, Holroyd CB, Yeung N (2005) Activity in human reward-sensitive brain areas is strongly context dependent. Neuroimage 25:1302-1309. CrossRef Medline

Padoa-Schioppa C (2009) Range-adapting representation of economic value in the orbitofrontal cortex. J Neurosci 29:14004-14014. CrossRef Medline

Padoa-Schioppa C, Assad JA (2008) The representation of economic value in the orbitofrontal cortex is invariant for changes of menu. Nat Neurosci 11:95-102. CrossRef Medline

Parducci A (1965) Category judgment: a range-frequency model. Psychol Rev 72:407-418. CrossRef Medline

Park SQ, Kahnt T, Rieskamp J, Heekeren HR (2011) Neurobiology of value integration: when value impacts valuation. J Neurosci 31:9307-9314. CrossRef Medline

Penny WD, Ridgway GR (2013) Efficient posterior probability mapping using Savage-Dickey ratios. PLoS One 8:e59655. CrossRef Medline

Petrovic P, Kalso E, Petersson KM, Ingvar M (2002) Placebo and opioid analgesia: imaging a shared neuronal network. Science 295:1737-1740. CrossRef Medline

Plassmann H, O’Doherty J, Rangel A (2007) Orbitofrontal cortex encodes willingness to pay in everyday economic transactions. J Neurosci 27: 9984-9988. CrossRef Medline

Plassmann H, O’Doherty JP, Rangel A (2010) Appetitive and aversive goal values are encoded in the medial orbitofrontal cortex at the time of decision making. J Neurosci 30:10799-10808. CrossRef Medline
Ploner M, Lee MC, Wiech K, Bingel U, Tracey I (2011) Flexible cerebral connectivity patterns subserve contextual modulations of pain. Cereb Cortex 21:719-726. CrossRef Medline

Rangel A, Clithero JA (2012) Value normalization in decision making: theory and evidence. Curr Opin Neurobiol 22:970-981. CrossRef Medline

Reid S, Wessely S, Crayford T, Hotopf M (2001) Medically unexplained symptoms in frequent attenders of secondary health care: retrospective cohort study. Br Med J 322:767. CrossRef Medline

Rudebeck PH, Saunders RC, Prescott AT, Chau LS, Murray EA (2013) Prefrontal mechanisms of behavioral flexibility, emotion regulation and value updating. Nat Neurosci 16:1140-1145. CrossRef Medline

Schoenbaum G, Takahashi Y, Liu TL, McDannald MA (2011) Does the orbitofrontal cortex signal value? Ann N Y Acad Sci 1239:87-99. CrossRef Medline

Seymour B, McClure SM (2008) Anchors, scales and the relative coding of value in the brain. Curr Opin Neurobiol 18:173-178. CrossRef Medline

Seymour B, O’Doherty JP, Koltzenburg M, Wiech K, Frackowiak R, Friston K, Dolan R (2005) Opponent appetitive-aversive neural processes underlie predictive learning of pain relief. Nat Neurosci 8:1234-1240. CrossRef Medline

Seymour B, Daw ND, Roiser JP, Dayan P, Dolan R (2012) Serotonin selectively modulates reward value in human decision-making. J Neurosci 32:5833-5842. CrossRef Medline

Soltani A, De Martino B, Camerer C (2012) A range-normalization model of context-dependent choice: a new model and evidence. PLoS Comput Biol 8:e1002607. CrossRef Medline

Stewart N, Brown GD, Chater N (2005) Absolute identification by relative judgment. Psychol Rev 112:881-911. CrossRef Medline

Talmi D, Dayan P, Kiebel SJ, Frith CD, Dolan RJ (2009) How humans integrate the prospects of pain and reward during choice. J Neurosci 29: 14617-14626. CrossRef Medline

Tremblay L, Schultz W (1999) Relative reward preference in primate orbitofrontal cortex. Nature 398:704-708. CrossRef Medline

Tzourio-Mazoyer N, Landeau B, Papathanassiou D, Crivello F, Etard O, Delcroix N, Mazoyer B, Joliot M (2002) Automated anatomical labeling of activations in SPM using a macroscopic anatomical parcellation of the MNI MRI single-subject brain. Neuroimage 15:273-289. CrossRef Medline

Vlaev I, Seymour B, Dolan RJ, Chater N (2009) The price of pain and the value of suffering. Psychol Sci 20:309-317. CrossRef Medline

Vlaev I, Chater N, Stewart N, Brown GD (2011) Does the brain calculate value? Trends Cogn Sci 15:546-554. CrossRef Medline

Vlaev I, Seymour B, Chater N, Winston JS, Yoshida W, Wright N, Symmonds M, Dolan R (2014) Prices need no preferences: social trends determine decisions in experimental markets for pain relief. Health Psychol 33:6676. CrossRef Medline

Wager TD, Rilling JK, Smith EE, Sokolik A, Casey KL, Davidson RJ, Kosslyn SM, Rose RM, Cohen JD (2004) Placebo-induced changes in FMRI in the anticipation and experience of pain. Science 303:1162-1167. CrossRef Medline

Wager TD, Atlas LY, Lindquist MA, Roy M, Woo CW, Kross E (2013) An fMRI-based neurologic signature of physical pain. N Engl J Med 368: 1388-1397. CrossRef Medline

Watkinson P, Wood AM, Lloyd DM, Brown GD (2013) Pain ratings reflect cognitive context: a range frequency model of pain perception. Pain 154: 743-749. CrossRef Medline

Weiskopf N, Hutton C, Josephs O, Deichmann R (2006) Optimal EPI parameters for reduction of susceptibility-induced BOLD sensitivity losses: a wholebrain analysis at $3 \mathrm{~T}$ and 1.5 T. Neuroimage 33:493-504. CrossRef Medline

Weiskopf N, Sitaram R, Josephs O, Veit R, Scharnowski F, Goebel R, Birbaumer N, Deichmann R, Mathiak K (2007) Real-time functional magnetic resonance imaging: methods and applications. Magn Reson Imaging 25:989-1003. CrossRef Medline

Wiech K, Kalisch R, Weiskopf N, Pleger B, Stephan KE, Dolan RJ (2006) Anterolateral prefrontal cortex mediates the analgesic effect of expected and perceived control over pain. J Neurosci 26:11501-11509. CrossRef Medline

Wiech K, Ploner M, Tracey I (2008) Neurocognitive aspects of pain perception. Trends Cogn Sci 12:306-313. CrossRef Medline

Winston JS, O’Doherty J, Kilner JM, Perrett DI, Dolan RJ (2007) Brain systems for assessing facial attractiveness. Neuropsychologia 45:195-206. CrossRef Medline

Yarkoni T, Poldrack RA, Nichols TE, Van Essen DC, Wager TD (2011) Large-scale automated synthesis of human functional neuroimaging data. Nat Methods 8:665-670. CrossRef Medline 